\title{
LLIO: Lightweight Learned Inertial Odometer
}

This paper was downloaded from TechRxiv (https://www.techrxiv.org).

\section{LICENSE}

CC BY 4.0

SUBMISSION DATE / POSTED DATE

23-08-2021 / 24-08-2021

CITATION

Wang, Yan; Kuang, Jian; niu, xiaoji (2021): LLIO: Lightweight Learned Inertial Odometer. TechRxiv. Preprint. https://doi.org/10.36227/techrxiv.16408383.v1

$\mathrm{DOI}$

10.36227/techrxiv.16408383.v1 


\title{
LLIO: Lightweight Learned Inertial Odometer
}

\author{
Yan Wang, Jian Kuang, and Xiaoji Niu
}

\begin{abstract}
The 3D position estimation of pedestrians is a vital module to build the connections between persons and things. The traditional gait model-based methods cannot fulfill the various motion patterns. And the various data-driven-based inertial odometry solutions focus on the $2 \mathrm{D}$ trajectory estimation on the ground plane, which is not suitable for AR applications. TLIO (Tight Learned Inertial Odometry) proposed an inertialbased 3D motion estimator that achieves very low position drift by using the raw IMU measurements and the displacement predict coming from a neural network to provide low drift pedestrian dead reckoning. However, TLIO is unsuitable for mobile devices because it is computationally expensive. In this paper, a lightweight learned inertial odometry network (LLIONet) is designed for mobile devices. By replacing the network in TLIO with the LLIO-Net, the proposed system shows similar accuracy but significantly improved efficiency. Specifically, the proposed LLIO algorithm was implemented on mobile devices and compared the efficiency with TLIO. The inference efficiency of the proposed system is 2-12 times that of TLIO. Source code can be found on this page:

https:// ....
\end{abstract}

Index Terms-Pedestrian Dead Reckoning (PDR), Inertial State Estimation, AI-Based Methods, Internet of Things (IoT)

\section{INTRODUCTION}

Augmented reality (AR) shows tremendous potential to make life more convenient. In AR, a pedestrian positioning system that can provide high accuracy $3 \mathrm{D}$ trajectories in indoor and outdoor environments plays a crucial role in connecting AR devices to the internet of things (IoT) network.

Various techniques were adopted to achieve indoor navigation in recent years. The positioning system based on Bluetooth low-energy (BLE) [1] and WIFI [2] [3] [4] can only achieve low accuracy positioning. The ultra-wideband (UWB) [5] can provide decimeter-level positioning accuracy in theory, but its performance significantly degrades in non-line-of-sight (NLOS) environments. Furthermore, both of the techniques mentioned above relied on pre-installed infrastructures.

Vision-based systems, such as the visual-inertial navigation system, have seen tremendous success today. The visualinertial navigation system (VINS) [6] [7] can achieve high accuracy positioning over a long period through combined vision and inertial measures. Meanwhile, the hardware cost of visual-inertial navigation systems has become acceptable

This work was supported in part by the National Key Research and Development Program of China (Grant No.2016YFB0502202) and the Natural Science Foundation of Hubei Province ( Grant No. 2020CFB230). (Corresponding author: Jian Kuang)

$\mathrm{X}$. Niu is with the GNSS Research Center, Wuhan University, Wuhan, Hubei, CO 430072 PR China. He is also with the Artificial Intelligence Institute of Wuhan University. (e-mail:xjniu@whu.edu.cn)

J. Kuang and Y. Wang are with the GNSS Research Center, Wuhan University, Wuhan, Hubei, CO 430072 PR China (e-mails: kuang@whu.edu.cn; wystephen@whu.edu.cn) for consumer-level devices. For these advantages, the VINS has been one of the best indoor positions, especially for AR. Despite the impressive performance of state-of-the-art VINS solutions, applying these methods in product scenarios is still facing some challenges. For example, the visionbased system relies heavily on consistent feature association. However, the feature association cannot be achieved in some challenging scenarios (such as positioning in a dark room or when the camera is blocked by obstacles). So, a system that can provide consistent pose estimation independent of external environments is necessary.

Inertial measurement unit (IMU) collects linear acceleration (accelerometer) and angle rate(gyroscope), which are used in the inertial navigation system (INS) for estimating 3D motion. The INS is a fully self-contained positioning system. In other words, the INS estimates trajectory without any dependency on the external environment. This feature indicated that INS is a good complement for the visual-based system in AR. In fact, INS is utilized widely in mobile devices which need indoor positioning. However, the MEMS IMU embedded in mobile devices, include mobile phones and AR headsets, cannot provide long-term motion estimation alone. Because the noise of low-end IMU is strong and the position accumulation error of strapdown INS is proportional to the square of time.

Pedestrian Dead Reckoning (PDR) uses sensors in mobile devices to detect the gait information and form a deadreckoning model. Some approaches [8] used prior knowledge of human motion to eliminate accumulation error of velocity. One way to applying the prior knowledge is detecting gait cycles and using this information to estimating trajectories. However, this way consists of several sub-modules: step detection, step length estimation, step orientation estimation. Each module needs several hand-designed rules or machine learning. For hand-designed rules, it is hard to find out rules suitable for every scenario and for all users. For machine learning, it is not easy to collect massive datasets with the ground truth for some sub-modules, e.g., step detection.

Recent research shows that data-driven inertial odometers can directly provide trajectories by integrating the average velocity through machine learning. Many approaches focus on achieving 2D positioning [9] [10] [11] [12]. IONet [11] first proposed an LSTM-based architecture to estimate relative displacement in the ground plane. RoNIN [10] assumption that the global orientation is calculated through fusing linear acceleration, angle rate, and magnetic. Then, estimate velocity through a neural network (including ResNet, LSTM, and TCN) based on acceleration and gyroscope represented in the gravity-aligned frame. IDOL [12] uses acceleration, angle rate, and magnetic to estimate global orientation rather than use global orientation estimated by traditional way. It shows the best accuracy on both orientation and position. Compare 
to traditional PDR, these learned-based methods show higher accuracy and are more robust for various motion patterns.

However, for AR headsets in complex environments, a 3D pose estimator is necessary. Tight learned interial odometry (TLIO) [13] achieve learned inertial odometry for AR headsets which can estimate 3D poses accuracy for complex scenarios. It adopts ResNet to estimate 3D displacement in short periods and uses Kalman Filter to fuse it with IMU measurements to achieve long-term dead reckoning. It shows the best performance in field testing but is computationally expensive. However, computational efficiency is a vital metric for AR applications run on mobile devices because the computation power of mobile devices is limited. In reality, the efficiency bottleneck of TLIO is the ResNet-based neural network which uses to inference 3D displacement. More specifically, the ResNet-based architecture adopted in TLIO is computation expensive and not friendly for mobile devices code implementations.

The recent multilayer perceptron (MLP) models [14] [15] [16] show potential to replace the ResNet because these architectures can provide better efficiency and accuracy trade off. For example, the recent MLP-based models can provide better efficiency with similar accuracy in image classification. Moreover, the MLP architecture mainly uses matrix multiplication which has been highly optimized in mobile devices readily. This fact indicated that the MLP architecture could be easily implemented on mobile devices

In this paper, we proposed a lightweight learned inertial odometry for mobile devices. It can provide low drift 3D dead reckoning on mobile devices with a low computational load. This paper has two major contributions:

- We proposed a lightweight MLP-based network to regression both the 3D displacement and the corresponding covariance. Specifically, we use this network to replace the ResNet architecture in TLIO and evaluate the system performance. The proposed networks provide similar performance and $(1.9$ - 12.0)x faster than ResNet-based methods when implementing on mobile devices.

- We conduct systematic research into the relationship between the computational efficiency and the positioning performance of the neural network models on mobile devices.

The rest of the paper is organized as follows. Section $I \mathrm{I}$ gives a brief description of the whole system. Section III describes the whole solution in details. Section [V] uses real test datasets to prove that the proposed network shows similar accuracy and significantly improved efficiency. Section $\mathrm{V}$ summarizes the whole paper.

We denoted the proposed system as the LLIO and the lightweight MLP-based network as LLIO-Net for further description in this paper.

\section{SySTEM OVERVIEW}

The proposed system uses raw IMU measurements (linear acceleration and angle rate) and provides 3D motion estimation related to the first instance. As shown in Fig 1 . this system consists of two components: a stochastic clone

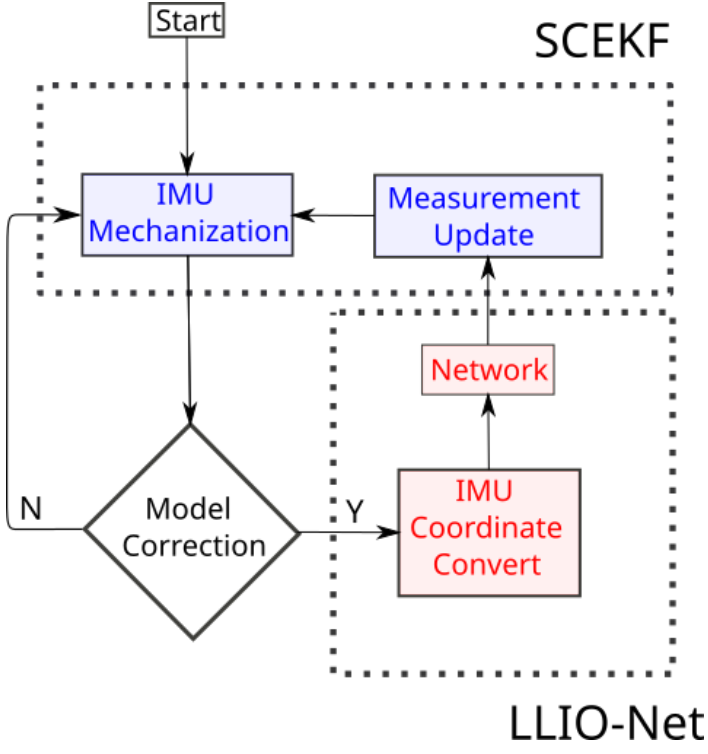

Fig. 1. System flowchart. The block named model correction selects keyframe for inference displacement based on LLIO-Net. In this paper, the key frame selected every $0.1 \mathrm{~s}$.

Extended Kalman Filter (SCEKF) [17] and a lightweight inertial odometry neural network (denoted as LLIO-Net).

The SCEKF estimates the 3D motion (including position, orientation, and velocity) and the bias of IMU. The block IMU mechanization is the propagation of the SCEKF. It predicts the system state based on IMU raw measurements. The input of the measurement update of SCEKF is the 3D displacement provided by the LLIO-Net. In summary, the filter tightly couples the raw IMU measurement and the displacement, which provides by LLIO-Net to estimate 3D motion and the IMU bias.

The LLIO-Net takes a sequence of IMU measurements represented in a gravity-aligned frame to estimate the displacement between the first and end instances. In the IMU coordinate convert block, the IMU measurements are converted from the IMU frame to the navigation frame using the rotation matrix estimated by the SCEKF. The network block estimates the displacement and the corresponding covariance based on converted IMU measurements. The LLIO-Net is inferences every 0.1 seconds and uses 1 second of IMU measurements as inputs.

The IMU measurement is utilized twice in the whole system. Firstly, the raw IMU measurements input for the IMU mechanization to estimate the prior distribution of the system state. Secondly, in the measurement update, the displacement is estimated by the IMU measurements using to mitigate accumulation errors of SCEKF. The primary information source of the second one is the human motion patterns memoried in the LLIO-Net rather than the IMU measurement itself.

\section{Algorithm DESCRIPTION}

\section{A. Coordinate Definition}

In this paper, three coordinate frames are defined: the navigation frame denoted as $\mathbf{F}^{N}$, the $t$-th body frame denoted as 


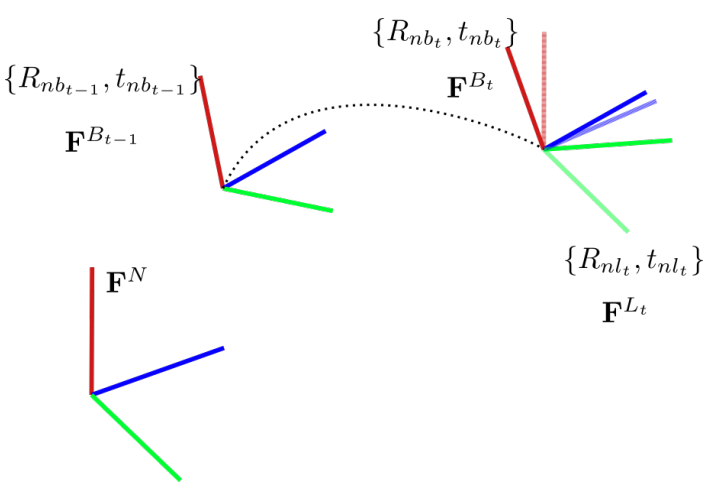

Fig. 2. Coordination Definition

$\mathbf{F}^{B_{t}}$, and the $t$-th local gravity-aligned coordinate denoted as $\mathbf{F}^{L_{t}} . \mathbf{F}^{B_{t}}$ aligned the coordinates of IMU at $t$ moments. $\mathbf{F}^{N}$ is a gravity-aligned coordinate. It is aligned with the IMU center at the initial moment. $\mathbf{F}^{L_{t}}$ is the gravity-aligned coordinate frame which the yaw is the same to $\mathbf{F}^{B_{t}}$. In this paper, the 3D motion in the SCEKF is parameterized as the position $\left(t_{n b_{t}}\right)$ of $t$-th body frame, rotation $\left(R_{n b_{t}}\right)$ from $t$-th body frame to the navigation frame, and the velocity of $t$-th body frame in the navigation frame. The IMU raw measurements at the $t$-th moments are denoted as $a_{t}^{B_{t}}$ and $\omega_{t}^{B_{t}}$ respectively. Moreover, the $a_{t}^{N}$ and $\omega_{t}^{N}$ denote them represented in the navigation frame.

\section{B. Lightweight Learned Inertial Odometry Network}

In this section, we intend to introduce the lightweight learned inertial odometer network (LLIO-Net). Figure 3 shows the framework of LLIO-Net.

1) Network architecture: The LLIO-Net uses a Residual Multi-Layer Perceptrons (ResMLP) architecture [15] as a feature extractor to predict displacement and corresponding covariance. Compared with the traditional MLP, the ResMLP use fewer parameters and can establish interactions between any two positions in the feature matrix, which the traditional MLP does. Compared with the ResNet, the ResMLP can achieve long-range interaction easier and with lower inductive bias. The proposed LLIO-Net consists of three modules. The feature convert module rearranges the raw input as a feature matrix. The ResMLP module extracts high-level features from the input feature embeddings. The regression module regresses displacement and corresponding covariance.

The feature convert module rearranged the IMU measurements in the navigation frame. Using the IMU measurements between $t-L$ and $t$ moments, the input is a $6 \times L$ matrix. Split the input into $N_{\text {patch }}$ patchs, each patch contained $L_{\text {feature }}$ measurements $\left(L=N_{\text {patch }} \times L_{\text {feature }}\right)$. Then flatten each patch and combined all features. After that, we got the $N_{\text {patch }}$ $\left(6 \times L_{\text {feature }}\right)$-dimensional embeddings. The resulting set of $N_{\text {patch }}$ embeddings are fed to a sequence of ResMLP block

The ResMLP module consists of a sequence of ResMLP blocks that all have the same structure. Before the ResMLP modules, a linear layers convert the $N_{\text {patch }}\left(6 \times L_{\text {feature }}\right)$ dimensional to the $N_{\text {patch }} L_{\text {feature }}^{\text {inner }}$-dimensional embeddings. The $L_{\text {feature }}^{i n n e r}$ is the feature dimensional in the ResMLP block.
Each ResMLP block is combined by affine layer (AFF), linear layer, and GELU layer (GELU).

The affine layer function as a alternation of the Layer Normalization. It simply rescales and shifts the input componentwise. More specifically, the affine layer is defined as:

$$
\operatorname{AFF}_{\alpha, \beta}(x)=\operatorname{Diag}(\alpha) x+\beta
$$

where $\alpha$ and $\beta$ are learnable vectors. Note, the $\operatorname{AFF}()$ to a matrix is applied independently to each column of the matrix.

Overall, the ResMLP is combined by the cross-patch interaction block and the cross-channel interaction block. The cross-patch interaction block is defined as:

$$
Z=M+\operatorname{AFF}\left(\left(A \operatorname{AFF}(M)^{T}\right)^{T}\right)
$$

The cross-channel interactions block is defined as:

$$
Y=Z+\operatorname{AFF}(C \operatorname{GELU}(B \operatorname{AFF}(Z)))
$$

where $A, B$ and $C$ are the main learnable parameters. The dimensions of the parameter matrix $A$ are $N_{\text {patch }} \times N_{\text {patch }}$. As a consequence, the dimensions of $Z$ same to $M$, is $N_{\text {patch }} \times\left(L_{\text {feature }}^{\text {inner }}\right)$. The dimensions of $B$ and $C$ are $L_{\text {feature }}^{\text {inner }} \times\left(E \times L_{\text {feature }}^{\text {inner }}\right)$ and $\left(E \times L_{\text {feature }}^{\text {inner }}\right) \times L_{\text {feature }}^{\text {inner }}$ respectively. Thus, the dimensions of $Y$ same to $Z$ and $M$. Here, the $E$ is the expansion dimension. Note, different from the original ResMLP, we added a dropout layer after the GELU. This block is not shown in Figure 3

The regression block uses the feature extracted from ResMLP module estimate two 3D vector: displacement $\hat{d}_{t}^{N}$ and the diagonal of the covariance matrix $\Sigma_{\hat{d}_{t}^{N}}$. We assumed that the uncertainty of $\hat{d}_{t}^{N}$ at each axis is independent to simplify the problem. Thus, the covariance matrix $\Sigma_{\hat{d}_{t}^{N}}$ is a diagonal matrix. The network structure is shown in Figure 3 consists of the average pooling layer, linear layer, and GELU layer.

2) Training Methonology: The LLIO-Net is trained based on two loss functions. The Mean Square Error (MSE) loss function for $\hat{d}_{t}^{N}$ and the Negative log-likelihood (NLL) loss function for $\hat{d}_{t}^{N}$ and $\Sigma_{\hat{d}_{t}^{N}}$ together. The MSE loss is defined as:

$$
\mathscr{L}_{M S E}(d, \hat{d})=\frac{1}{n} \sum\|d-\hat{d}\|^{2}
$$

where $d$ is the ground truth displacement and $\hat{d}$ is the estimated displacement of the network. Through minimize the $\mathscr{L}_{M S E}$, the network learned to estimate 3D displacement.

The NLL loss function is defined as:

$$
\mathscr{L}_{N L L}(d, \hat{d}, \hat{\Sigma})=\frac{1}{n} \sum \frac{1}{2} \log (\operatorname{det}(\hat{\Sigma}))+\frac{1}{2}\|d-\hat{d}\|_{\hat{\Sigma}}^{2}
$$

where $\hat{\Sigma}$ is the corresponding covariance matrix of $\hat{d}$. And $\|d-\hat{d}\|_{\hat{\Sigma}}^{2}$ defined as

$$
\|d-\hat{d}\|_{\hat{\Sigma}}^{2}=(d-\hat{d})^{T} \hat{\Sigma}(d-\hat{d})
$$

. Through minimize the $\mathscr{L}_{N L L}$, the network learned to estimate the covariance correspond to the 3D displacement.

During the training stage, the $\mathscr{L}_{M S E}$ is adopted first until the network converges. Then, use the $\mathscr{L}_{N L L}$ to training $\hat{\Sigma}$ and $\hat{d}$ together until converges. 


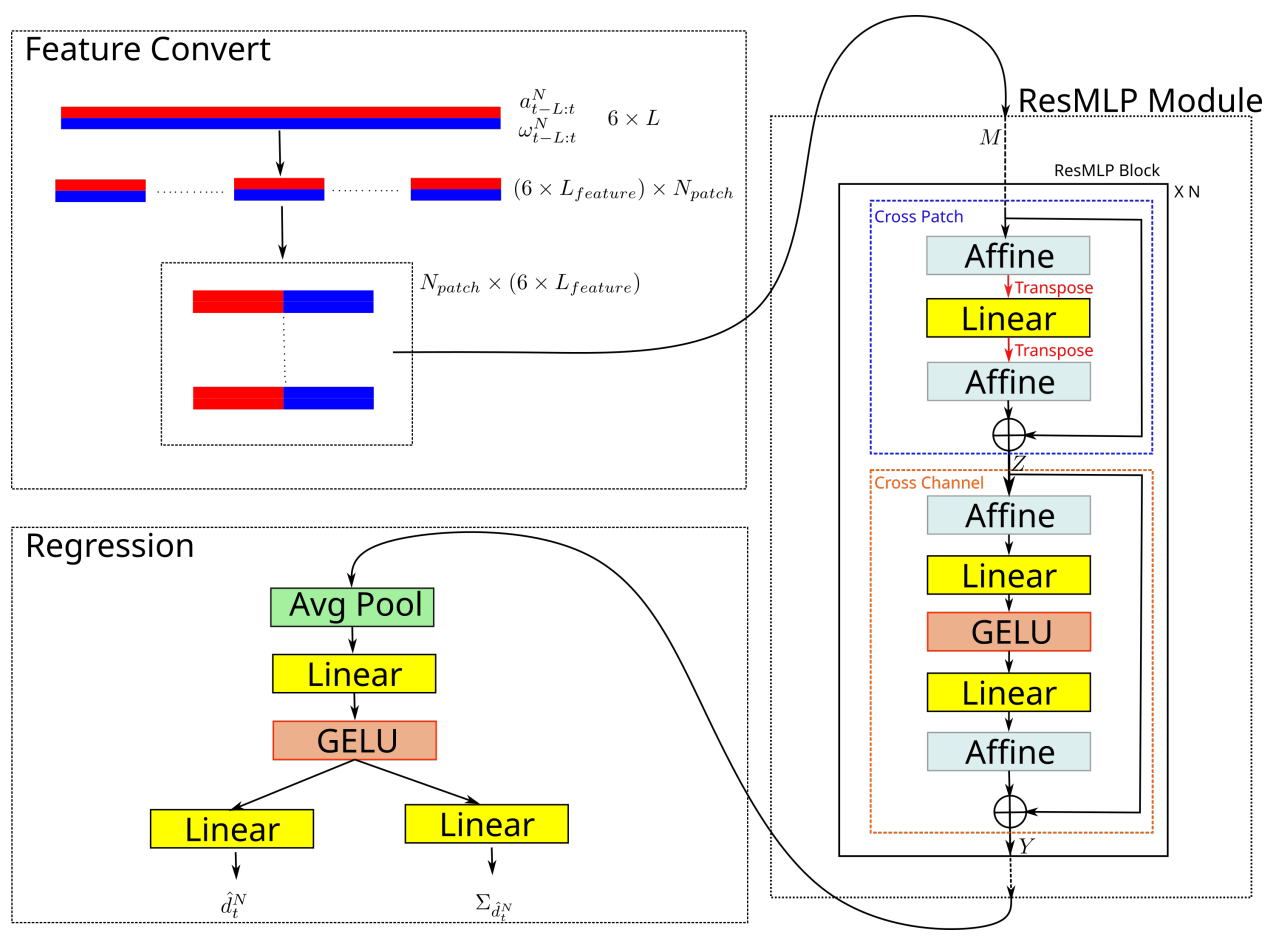

Fig. 3. The framework illustration of LLIO-Net.

\section{State Clone Extended Kalman Filter}

1) System State Definition: The full system state at $t$ moment is defined as:

$$
X_{t}=\left(s_{t}, \eta_{1}, \ldots, \eta_{m}\right)
$$

where $\eta$ are past system states, and $s_{t}$ is the current system state. More specifically,

$$
\begin{gathered}
\eta_{i}=\left[R_{n b_{i}}, t_{n b_{i}}\right], \\
s_{t}=\left[t_{n b_{t}}, R_{n b_{t}}, v_{n b_{t}}, b_{a}, b_{g}\right]
\end{gathered}
$$

We express $R_{n b_{t}}$ as the rotation from $\mathbf{F}^{B_{t}}$ to $\mathbf{F}^{N}$, and $t_{n b_{t}}$ and $v_{n b_{t}}$ are the position and velocity of $\mathbf{F}^{B_{t}}$ in the $\mathbf{F}^{N}$ respectively. $b_{a}$ and $b_{g}$ are the IMU accelerometer and gyroscope bias. Indeed, we use the IMU noise model defined as:

$$
\begin{aligned}
& a_{t}^{b_{t}}=\hat{a}_{t}^{b_{t}}+b_{a}+n_{a} \\
& \omega_{t}^{b_{t}}=\hat{\omega}_{t}^{b_{t}}+b_{g}+n_{g}
\end{aligned}
$$

$a_{t}^{b_{t}}$ and $\omega_{t}^{b_{t}}$ are measured value of acceleration and angle rate. $\hat{a}_{t}^{b_{t}}$ and $\hat{\omega}_{t}^{b_{t}}$ are true value of acceleration and angle rate. $n_{a}$ and $n_{g}$ are random noise variables following a zero-centered Gaussian distribution. Moreover, evolution of $b_{a}$ and $b_{g}$ are modeled as a discrete random walk processing.

The error-state-based indirect Kalman filter is utilized in the proposed system. The error state indicated the difference between estimated and real velue, estimated in the SCEKF. It is defined as:

$$
\begin{gathered}
\delta X_{t}=\left(\delta s_{t}, \delta \eta_{1}, \ldots, \delta \eta_{m}\right) \\
\delta s_{t}=\left[\delta t_{n b_{t}}, \phi_{n b_{t}}, \delta v_{n b_{t}}, \delta b_{a}, \delta b_{g}\right] \\
\delta \eta_{i}=\left[\delta t_{n b_{i}}, \phi_{n b_{i}}\right]
\end{gathered}
$$

Hence, the dimension of the system is $15+6 m$, where $m$ is the number of cloned system states and 15 is the dimension of $s_{t}$.

Since the rotation cannot add directly, the error of rotation $\phi_{n b_{t}}$ is defined as:

$$
\hat{R}_{n b_{t}}=R_{n b_{t}} \exp _{S O 3}\left(\phi_{n b_{t}}\right)
$$

$\hat{R}_{n b_{t}}$ and $R_{n b_{t}}$ represent estimate and real value of rotation respectively. $\exp _{\mathrm{SO} 3}(\cdot)$ denotes the $S O(3)$ exponential map.

2) State Propagation: The filter propagates the system state using the IMU raw measurements based on IMU mechanization. Since the proposed system aims to pedestrian motion estimation, the trajectory length is limited. Thus, we ignored the earth's curvature. Meanwhile, the gravity $g^{N}$ is assumed to be equal at every place of the navigation frame. The simplified strapdown IMU mechanization is defined as:

$$
\begin{gathered}
\hat{R}_{n b_{t}}=\hat{R}_{n b_{t-1}} \exp _{S O 3}\left(\left(\hat{\omega}_{t}^{B_{t}}-b_{g}\right) \Delta t\right) \\
\hat{v}_{n b_{t}}=\hat{v}_{n b_{t-1}}+g^{N} \Delta t+\hat{R}_{n b_{t}}\left(\hat{a}_{t}-b_{a}\right) \Delta t \\
\hat{t}_{n b_{t}}=\hat{t}_{n b_{t-1}}+0.5\left(\hat{v}_{n b_{t-1}}+\hat{v}_{n b_{t}}\right) \Delta t
\end{gathered}
$$

The cloned states need not update in the propagation stage.

The error-state covariance propagation can be written as:

$$
\begin{gathered}
P_{t}=\Phi_{t} P_{t-1} \Phi_{t}^{T}+G_{t} Q G_{t}^{T} \\
\Phi_{t}=\left[\begin{array}{cc}
\Phi_{t}^{s} & 0 \\
0 & I_{6 m}
\end{array}\right], G_{t}=\left[\begin{array}{c}
G_{t}^{s} \\
0
\end{array}\right]
\end{gathered}
$$

where, $\Phi_{t}^{s}$ and $G_{t}^{s}$ are linearized state propagation matrix of previous state $\hat{s}_{t-1}$ and all noise (include sensor noise and bias random walk noise) respectively. $I_{6 m}$ is a $6 m$ dimension identity matrix. 
3) State Augmentation: The measurement update of the SCEKF using the relative position of current system state and a previous system state. Thus, the previous system state should be maintained in the SCEKF through the techniques named stochasitc clone. The cloned system state is directly a copy of the current system state (only $t_{n b_{t}}$ and $R_{n b_{t}}$ ). The probability propagation of the stochastic clone step in the proposed system is defined as:

$$
\begin{gathered}
P_{t}^{\text {new }}=\left[\begin{array}{ccc}
I_{15} & 0 & 0 \\
0 & I_{6 m} & 0 \\
A & 0 & 0
\end{array}\right] P_{t}\left[\begin{array}{ccc}
I_{15} & 0 & 0 \\
0 & I_{6 m} & 0 \\
A & 0 & 0
\end{array}\right]^{T} \\
A=\left[\begin{array}{ccc}
I_{3 \times 3} & 0_{3 \times 3} & 0_{3 \times 9} \\
0_{3 \times 3} & I_{3 \times 3} & 0_{3 \times 9}
\end{array}\right]
\end{gathered}
$$

4) Measurement Update: The LIO-Net provides the pseudo measurement uses the acceleration and angle rate in a gravityaligned coordinate frame. Thus, the outputted of LIO-Net is represented in these gravity-aligned frames. As described in Section II] each IMU measure uses 10 times as input of LIO-Net. Converting measurements at different moments to the same coordinate frame can avoid redundant coordinate conversion. Thus, all IMU measurements were converted to the navigation frame, and the output of LIO-Net is represented in the navigation frame. However, the displacement in the navigation frame implicated a constrain to the absolute heading. More specifically, the displacement in the navigation frame leads to the absolute heading observable in the SCEKF. Nevertheless, the absolute heading is unobservable in theory. To mitigate this problem, the 3D displacement was converted to the local gravity-aligned frame $F^{L_{t}}$, anchored to the $t$-th body frame $F^{B_{t}}$.

The displacement and its covariance, which the LIO-Net outputts, are represented in $F^{N}$ denoted as $\hat{d}_{t}^{N}$ and $\Sigma_{\hat{d}_{t}^{N}}$ respectively. And the information used in the measurement update is defined as:

$$
\begin{gathered}
\hat{d}_{t}^{L_{t}}=\hat{R}_{\text {yaw }_{t}}^{T} \hat{d}_{t}^{N} \\
\Sigma_{\hat{d}_{t}^{L_{t}}}=\hat{R}_{\text {yaw }_{t}}^{T} \Sigma_{\hat{d}_{t}^{N}} \hat{R}_{\text {yaw }_{t}}
\end{gathered}
$$

The $\hat{R}_{\text {yaw }_{t}}$ is the heading rotation matrix of $\hat{R}_{n b_{t}}$. More specifically, the $\hat{R}_{n b_{t}}$ can be decomposed to three roation matrix $\left(\hat{R}_{n b_{t}}=\hat{R}_{\text {yaw }_{t}} \hat{R}_{\text {pitch }_{t}} \hat{R}_{\text {roll }_{t}}\right)$, where $\hat{R}_{\text {yaw }_{t}}, \hat{R}_{\text {pitch }_{t}}$, and $\hat{R}_{\text {roll }_{t}}$ corresponds to yaw, pitch and roll respectively.

Hence, the measurement function can be written as:

$$
h\left(X_{t}\right)=R_{\text {yaw }_{t}}^{T}\left(t_{n b_{j}}-t n b_{i}\right)=\hat{d}_{t}^{L_{t}}+n_{\hat{d}_{t}^{L_{t}}}
$$

where, $t_{n b_{j}}$ represent the position of $j$-th moment. In this paper, $j$-th moment are 1 second before $i$-th moment. $n_{\hat{d}_{t}^{L_{t}}}$ follows the normal distributed $\mathcal{N}\left(0, \Sigma_{\hat{d}_{t}^{L_{t}}}\right)$.

In practice, there are some abnormal pseudo observations contained in the LIO-Net outputs. To mitigate the effect of abnormal observations, a $\chi^{2}$-test is employed. In detail, the observation checked by the following condition:

$$
\left\|R_{y^{T a w_{t}}}^{T}\left(t_{n b_{j}}-t_{n b_{i}}\right)-\hat{d}_{t}^{L_{t}}\right\|_{H P H+\Sigma_{\hat{d}_{t}^{L} t}}<\alpha
$$

$H$ is the jacobian matrix of $h\left(X_{t}\right) . \alpha$ is the threshold of $\chi^{2}$ test. We chooses $\alpha=11.345$ correponds to the $99 \%$ of the $\chi^{2}$ distribution with 3 degree of freedom. Furthermore, to avoid continuous reject pseudo observable leads to SCEKF crashed, we direct accept the LLIO-Net output if the previous three are rejected.

\section{EXPERIMENTS}

In this section, we compared our proposed LLIO-Net with TLIO in the scenario of a head-mounted AR device. We denote the ResNet architecture in TLIO as ResNet for further description. Furthermore, we compared three versions of LLIO-Net denoted as ResMLP512, ResMLP256, and ResMLP512. All metrics are compared on the test set, which is never present in the training stage. Note, all method uses the same setup except hyperparameters for defines networks and trains networks.

This section is organized as follows. Section IV-A describes implementation details. Section IV-B describes metrics for evaluate accuracy of estimated trajectories. Section IV-C compares the accuracy of all methods. Section IV-D analysis the inference efficiency of the proposed methods.

\section{A. Setup}

1) Data Preparation: The dataset is collected by the Asus tango phone, which is widely used in the domain of datadriven inertial odometry. The Asus tango phone can estimate 3D motion through a fisheye global shutter camera and embedded IMU module based on the visual-inertial odometry technique. In our experiments, we close the trigger of area learning to obtain a smooth trajectory. The trajectory output by the visual-inertial odometry function as ground truth in both training and evaluation stages. The full dataset contains more than 40 hours of head-mounted pedestrian data, including various activities (walking, standing still, sitting down and up, going down and up the stairs). The datasets are captured by six people with multiple different physical devices. Thus, the dataset contains a wide range of individual motion patterns and IMU systematic errors. Since in the AR applications, this ground truth is easy to collect by AR device itself. We follow the data split method of TLIO, split the dataset into $80 \%$ training, $10 \%$ validation, and $10 \%$ testing subsets randomly.

2) Data Augmentation Strategy: As described before, we use $L$ IMU samples IMU as input for the network. In this paper, we select $L=100$ as we collect IMU at $100 \mathrm{~Hz}$. Thus, each moment we send 1 second of IMU samples to the network. We first convert all IMU measurements from the body frame to the navigation frame based on the ground truth rotation matrix in the training stage. Moreover, the following data augmentation strategies are adopted to improve the generalization of the network:

- Use a random horizontal rotation to IMU measurements and displacement together. $\left([-180,180]^{\circ}\right)$. Because we assumed the AR headset could be oriented to arbitrary heading with respect to the walking direction.

- Add a random bias to IMU measurements $\left([-0.2,0.2] \mathrm{m} / \mathrm{s}^{2}\right.$ and $\left.[-0.5,0.5]^{\circ} / \mathrm{s}\right)$, to make the network robust to the IMU bias.

- Add a random perturbation of gravity direction $\left([0,5]^{\circ}\right)$ to make the network robust to the gravity orientation perturbation. 
3) SCEKF Setting: In this paper, we use measurements update at $10 \mathrm{~Hz}$ and use 1 second of IMU samples to estimate displacement as described before. Thus, the SCEKF contained 9 cloned states in the filter. Furthermore, SCEKF will remove the last cloned state immediately after the measurement update. In the evaluation stage, the SCEKF needs to be initializing. We initialize the SCEKF based on the rotation matrix at the first moment only to simulate the scenario the IMU system should be working individually. This way is reasoning because the rotation could be provided by an AHRS system easily. The bias of IMU is set to zero as the initial value and estimate online.

4) Training Details: We implemented all models by PyTorch 1.8 framework [18]. The ResNet uses hyperparameters provided in TLIO [13]. Those hyperparameters show the most outstanding performance in our dataset too. The proposed ResMLP series model uses 6 ResMLP blocks, 0.2 dropout probability. The patch length $L_{\text {patch }}$ is 25 . The total length $L$ is 100 for 1 second IMU measurements collected at $100 \mathrm{~Hz}$. The inner feature dimensional $L_{\text {feature }}^{\text {inner }}$ of ResMLP is 512, 256, and 128 for ResMLP512, ResMLP256, and ResMLP512 respectively. All models are trained via an ADAM optimizer [19]. The ResNet uses a learning rate of 1e-4. Moreover, the ResMLP series uses a learning rate of $5 \mathrm{e}-4$. We trained each of the model configurations on the NVIDIA GTX 3090.

\section{B. Evaluation Metric}

To evaluate the positioning performance, we define the following metrics similar to TLIO:

- $\operatorname{ATE}(\mathrm{m}): \sqrt{\frac{1}{n} \sum\left\|t_{n b_{t}}-\hat{t}_{n b_{t}}\right\|^{2}}$

$t_{n b_{t}}$ and $\hat{t}_{n b_{t}}$ are ground truth position and estimated position at $t$ moment respectively. The absolute translation error (ATE) computed as root-mean square error (RMSE) of the esimated trajectory and the ground true trajectory.

- RTE- $\Delta t(\mathrm{~m})$ :

$\sqrt{\frac{1}{n} \sum\left\|R_{\text {yaw }}^{T}\left(t_{n b_{t}}-t_{n b_{t-\Delta t}}\right)-\hat{R}_{\text {yaw }}^{T}\left(\hat{t}_{n b_{t}}-\hat{t}_{n b_{t-\Delta t}}\right)\right\|^{2}}$ $R_{\text {yaw }}$ and $\hat{R}_{\text {yaw }}$ are the groud truth and estimated heading rotation matrix respectively. The relative translation error (RTE) is error of the trajectory represented in the local gravity-aligned frame at $t-\Delta t$ moment. Hence, the RTE is not affected by the global yaw drift. In our implementation, the $\Delta t=1$ minute.

- RTE- $\Delta L(\mathrm{~m})$ :

$\sqrt{\frac{1}{n} \sum\left\|R_{y a w}^{T}\left(t_{n b_{t}}-t_{n b_{k}}\right)-\hat{R}_{y a w}^{T}\left(\hat{t}_{n b_{t}}-\hat{t}_{n b_{k}}\right)\right\|^{2}}$

RTE- $\Delta L$ is the relative translation error (RTE) for every $10 \mathrm{~m}$ trajectory $(\Delta L=10 \mathrm{~m})$.

- $\operatorname{AYE}\left(^{\circ}\right): \sqrt{\frac{1}{n} \sum\left\|\gamma_{t}-\hat{\gamma}_{t}\right\|^{2}}$

$\gamma_{t}$ and $\hat{\gamma}_{t}$ are heading orientation of ground truth and estimated trajectory. The abolute yaw error (AYE) is the RMSE of abolute heading drift. In practical, we calculation rotation difference through three-axis rotation matrix and decompose the yaw component to calculate AYE.

- RYE- $\Delta t \quad\left(^{\circ}\right): \sqrt{\frac{1}{n} \sum\left\|\left(\gamma_{t+\Delta t}-\gamma_{t}\right)-\left(\hat{\gamma}_{t+\Delta t}-\hat{\gamma}_{t}\right)\right\|^{2}}$ The relative yaw error (RYE) is calculated in the same way of AYE. $\Delta t=1$ miniutes.

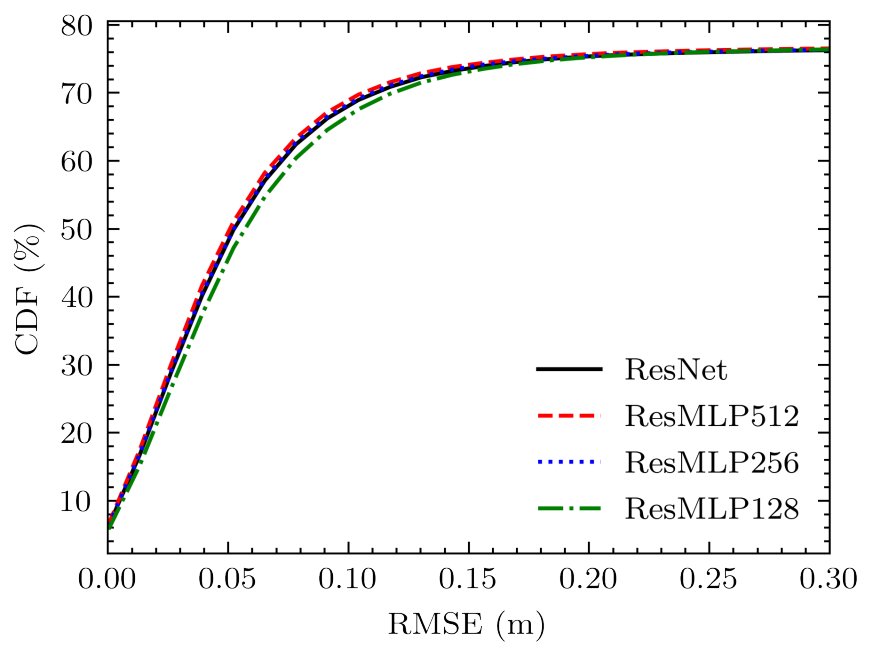

Fig. 4. A Comparison of inference error CDF of different models in test set. The network inferences displacement in 1 second as described before.

\section{System Performance}

We conducted a systematic comparison of the ResNet and ResMLP series in this section. In detail, we compared the inference accuracy of the network and positioning accuracy of the whole system uses different networks.

Table I gives an overview of the performance comparison. The distance error in the Table I means the average error of inference results defined as $\frac{1}{n} \sqrt{\sum\left\|t_{n b_{t}}-\hat{t}_{n b_{t}}\right\|^{2}}$ which is slightly different from the RMSE. This metric is used to directly evaluate neural network performance. And Figure 4 gives the cumulative distribution function (CDF) of inference RMSE. Obviously, the ResMLP series show similar inference performance for both average distance error and CDF of inference RMSE. Specifically, the ResMLP512 and ResMLP256 show slightly better inference accuracy to ResNet, and the ResMLP128 is slightly worse than ResNet.

Table I] also provides ATE, RTE- $\Delta t$, RTE- $\Delta L$, AYE, and RYE- $\Delta t$ of trajectories uses different networks. Note, all the relative metrics use a sliding window to calculate those metrics. The step length of sliding windows is $\frac{1}{10}$ of the sliding window length(etc. $6 \mathrm{~s}$ for TRTE- $\Delta t$ ). Even ResMLP512 showed slightly better RTE- $\Delta t$, RTE- $\Delta L$, and RYE than ResNet; the ResNet showed better ATE and AYE. Since those trajectories are collected over 15 minutes, the AYE and ATE are easily to be effect by random perturbation. Figure 5 shows CDF of RTE- $\Delta t$, RTE- $\Delta L$, and RYE- $\Delta t$. All methods show the same level of accuracy except ResMLP128. The ResMLP128 shows slightly worse performance than ResNet in the point of view of stochastic metrics.

Meanwhile, we give a group of selected trajectories to compare the positioning performance. Figure 6 shows a selection of trajectories to illustrate the performance of trajectories with different contours. All methods work well when the pedestrian walks straight and worsens when the pedestrian stands still or walks around a small area for the long term. Figure 7 shows a 3D trajectory. All methods can rightly estimate trajectory when going up and down stairs. 
TABLE I

PERFORMANCE COMPARISON BETWEEN RESNET BASED SOLUTION AND THE PROPOSED LLIO-NET.

\begin{tabular}{ccccccc}
\hline Model & Distance Error $(\mathrm{m})$ & ATE $(\mathrm{m})$ & RTE- $\Delta t(\mathrm{~m})$ & RTE- $\Delta L(\mathrm{~m})$ & AYE $\left({ }^{\circ}\right)$ & RYE- $\Delta t\left(^{\circ}\right)$ \\
\hline ResNet & 0.111 & 6.68 & 0.900 & 0.239 & 11.54 & 2.46 \\
ResMLP512 & 0.108 & 7.40 & 0.884 & 0.237 & 12.99 & 2.39 \\
ResMLP256 & 0.110 & 6.80 & 0.963 & 0.255 & 11.48 & 2.36 \\
ResMLP128 & 0.119 & 7.51 & 1.10 & 0.286 & 13.1 & 2.44 \\
\hline
\end{tabular}

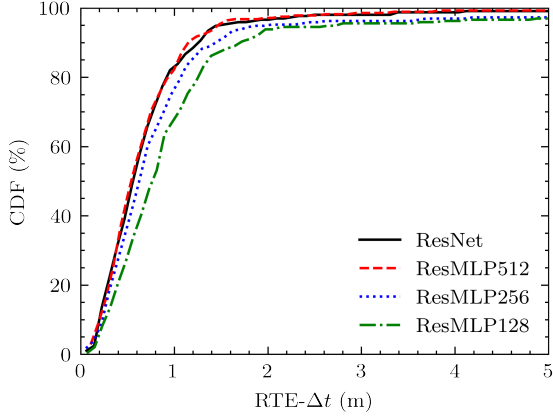

(a)

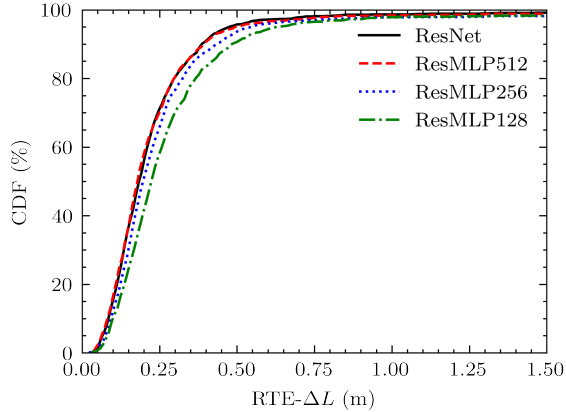

(b)

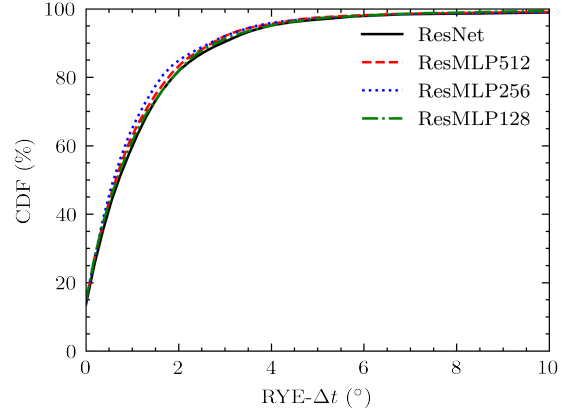

(c)

Fig. 5. CDF of relative error metrics of different methods in test set.

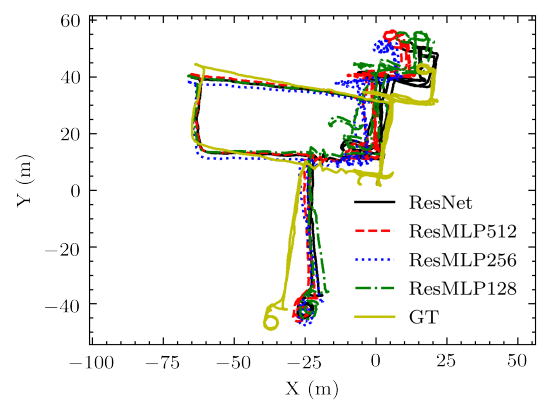

(a)

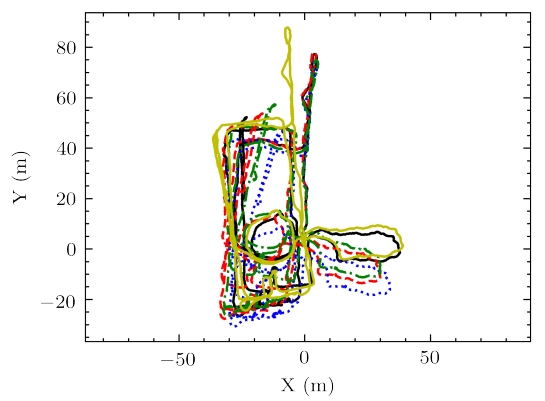

(d)

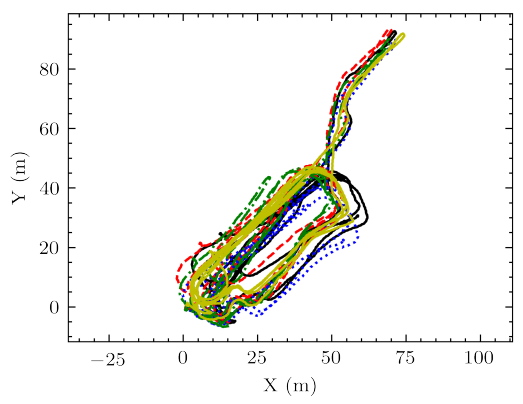

(b)

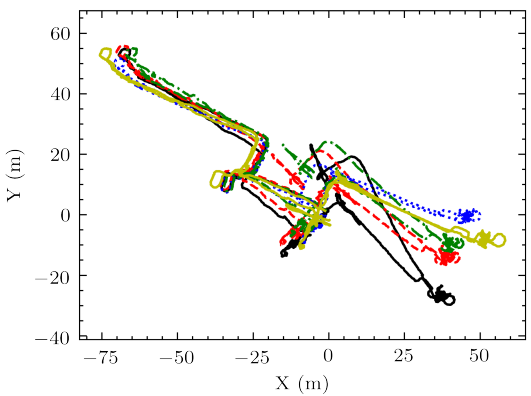

(e)

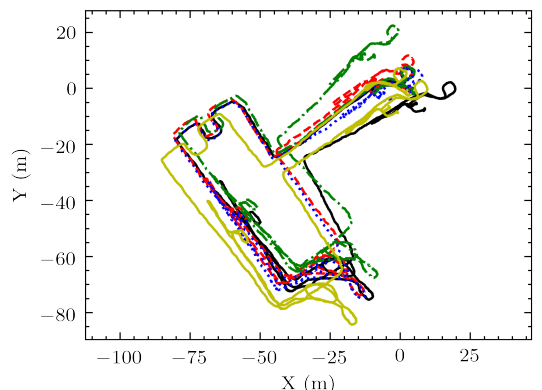

(c)

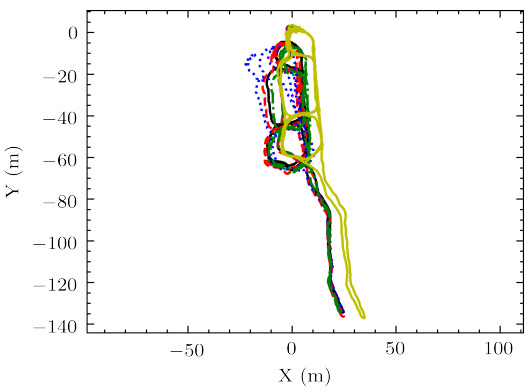

(f)

Fig. 6. Selection of trajectories with different contours. The ground truth trajectory generated from Tango phone denoted as GT marked as yellow. The ResNet trajectory is marked as the black line. The ResMLP512, ResMLP256, and ResMLP128 marked as red, blue and green respectively.

This section fully compared the performance of proposed networks and ResNet from the point of view of inference accuracy, stochastic absolute and relative metrics, and illustration of trajectories in 2D and 3D. In summary, ResMLP512 and ResMLP256 show similar performance to the ResNet. ResMLP128 is slightly worse than ResNet in all metrics but still shows the same level of performance in positioning. Furthermore, since the ground truth is used visual-inertial odometry without loop-closure, the ground truth trajectory shows a bit of cumulative positioning error as shown in Figure 6 We compared network performance in the next section based on inference accuracy and relative accuracy metrics to avoid the cumulative error.

\section{Inference Efficiency on mobile devices}

In the proposed system, the main computation cost is generated from two modules, the SCEKF (including propagation, state clone, and measurement update) and the network. We 
TABLE II

COMPARISON OF COMPUTATION EFFICIENCY

\begin{tabular}{|c|c|c|c|c|c|c|}
\hline Model & & & ResNet & ResMLP512 & ResMLP256 & ResMLP128 \\
\hline Distance Error (m) & & & 0.111 & 0.108 & 0.110 & 0.119 \\
\hline FLOPs (M) & & & 21.15 & 25.78 & 6.54 & 2.08 \\
\hline \multirow{6}{*}{ Inference Time (ms) } & \multirow{2}{*}{ Pixel 3} & JIT Model & $9.90(1 \mathrm{x})$ & $14.5(0.68 \mathrm{x})$ & $4.80(2.1 \mathrm{x})$ & $1.72(5.7 x)$ \\
\hline & & Mobile Model & $7.57(1 \mathrm{x})$ & $3.90(1.9 x)$ & $1.60(4.7 x)$ & $0.82(9.2 x)$ \\
\hline & \multirow{2}{*}{ Huawei Mate 30} & JIT Model & $7.41(1 \mathrm{x})$ & $8.05(0.92 x)$ & $2.42(3.1 \mathrm{x})$ & $0.978(7.5 x)$ \\
\hline & & Mobile Model & $6.11(1 \mathrm{x})$ & $2.10(2.9 \mathrm{x})$ & $0.851(7.2 \mathrm{x})$ & $0.503(12 x)$ \\
\hline & \multirow{2}{*}{ Huawei Mate 40} & JIT Model & $6.20(1 \mathrm{x})$ & $5.97(1.03 x)$ & $1.89(3.3 \mathrm{x})$ & $0.741(8.3 x)$ \\
\hline & & Mobile Model & $5.08(1 \mathrm{x})$ & $1.83(2.8 \mathrm{x})$ & $0.718(7.1 x)$ & $0.435(12 x)$ \\
\hline
\end{tabular}

(a)

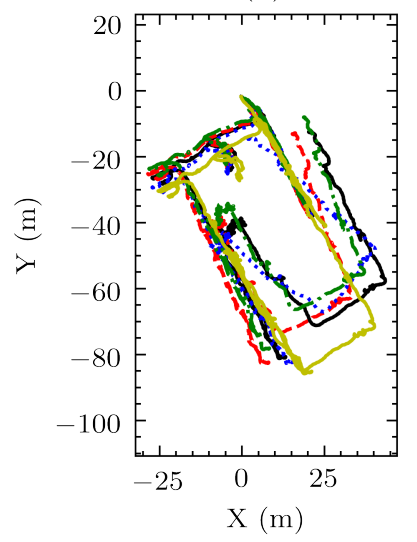

(b)

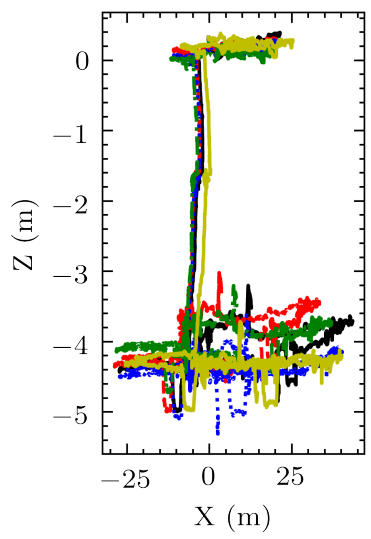

Fig. 7. Selected trajectory with height illustrated. (a) illustrated trajectory in $\mathrm{x}-\mathrm{y}$ plane. (b) illustrated trajectory in $\mathrm{x}-\mathrm{z}$ plane. The ground truth trajectory generated from the Tango phone is denoted as GT marked as yellow. The ResNet trajectory is marked as the black line. The ResMLP512, ResMLP256, and ResMLP128 are marked as red, blue, and green, respectively.

implement a C++ version of SCEKF to test the computational efficiency. The $\mathrm{C}++$ version of SCEKF can process data at 190X faster than in real-time (uses 2.9 seconds processing time for 561 seconds dataset). Meanwhile, the inferencing speed of networks is significantly lower than SCEKF. Thus, the efficiency bottleneck of this $3 \mathrm{D}$ inertial odometry is the network. Aim to implementation on the mobile devices, the efficiency of the network is systematically analyzed in this section.

To illustrated the computational efficiency, we compared the inference time in follows devices.

- Google Pixel 3 (announced 2018)

It equipped with octa-core CPU (Snapdragon $8454 \times 2.5$ $\mathrm{GHz} \& 4 \times 1.6 \mathrm{GHz}$ ).

- Huawei Mate 30 5G (announced 2019)

It equipped with octa-core CPU (Kirin 990 2x2.86 GHz \& $2 \times 2.36 \mathrm{GHz} \& 4 \times 1.95 \mathrm{GHz}$ ).

- Huawei Mate 40 (announced 2020)

It equipped with octa-core CPU(Kirin 9000 1.x3.13 GHz \& 3x2.54 GHz \& 4x2.05 GHz).

The mobile devices use PyTorch for Android 1.8.0 as a deep learning inference framework. Every model inference 1000 times uses CPU to calculate average inferences time. We tested two versions of the just-in-time (JIT) compilation model on mobile devices. The JIT Model converted uses the JIT compiler to convert the model based on JIT only. The Mobile

Model converted uses the JIT compiler and PyTorch builtin mobile optimizer. Moreover, the inference result of the JIT Model and the Mobile Model are compared. The difference of such results is smaller than 1e-14. Thus, the JIT Model and the Mobile Model can provide the same positioning performance.

Table [I] shows the computation efficiency comparison. The floating-point operations (Flops) of each model are provided. The inference time ratio compared to ResNet at each setup was also provided. The FLOPs of ResMLP256 and ResMLP128 are significantly less than the ResNet and show efficiency improvement uses both of JIT Model and Mobile Model. The ResMLP512 with more FLOPs than ResNet but shows better inference efficiency in testing the Mobile Model. This may benefit from the optimization strategy in the mobile optimizer of PyTorch. In detail, the ResMLP series shows better efficiency improvement on Mobile Model. The inference time of ResMLP256 is 4.7 - 7.2 times faster compared to the ResNet. Moreover, the ResMLP128 with slightly worse accuracy shows 9.2 - 12 times faster inference time.

To illustrate the relation between accuracy and efficiency, Figure 8 shows the relationship between inference time and RTE- $\Delta t$ of different models at Huawei Mate 30.

In summary, the ResMLP series shows a higher accuracyefficiency ratio than ResNet. Although all the models can run in real-time on current mobile devices, the computational cost is still a key metric to evaluate the algorithm that needs to be executed on mobile devices. The positioning algorithm usually functions as a fundamental of other applications and runs at the whole workflow. Thus the improvement of efficiency of ResMLP is vital in this scenario.

\section{CONCLusion}

In this paper, we proposed LLIO, a lightweight learned inertial odometry, which introducing the LLIO-Net to replace the ResNet-based architecture. The LLIO-Net module estimates 3D displacement and the corresponding covariance to eliminate the accumulation error of INS mechanization. The experiments proved that the proposed LLIO-Net could achieve the same level of accuracy compared with the TLIO but with significantly improved computational efficiency (up to $12 \mathrm{x}$ faster). The inference efficiency test on mobile devices shows that the proposed inertial odometry can be implemented on mobile devices and functions as a low drift pedestrian 3D motion estimator. Because of its low computational load and low drift, the LLIO can be adopted as a backup of visualinertial odometry in AR applications. Alternatively, it can 


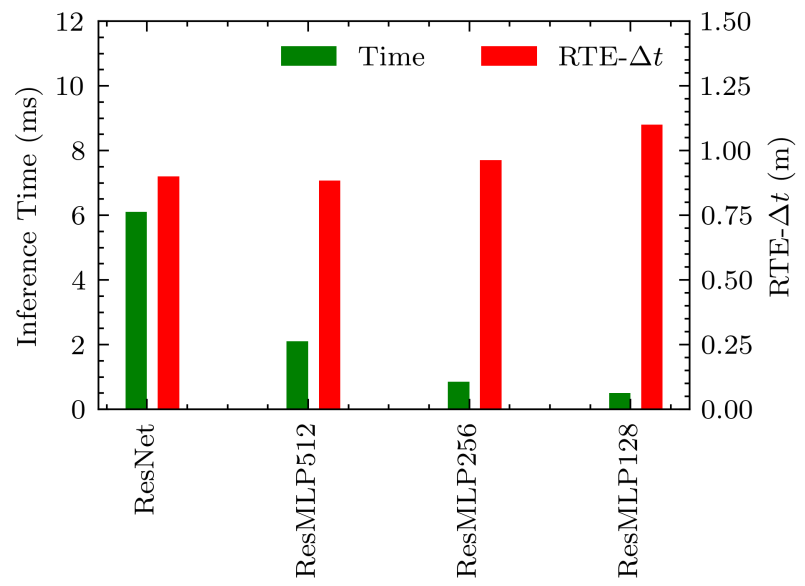

Fig. 8. Inference time and RTE- $\Delta t$ of mobile models on Mate 30.

function as the independent dead reckon algorithm for fusing other sources of information.

Further work would focus on the generalization of the model. For example, improve the performance for estimate 3D trajectories of pedestrians without labeled IMU sequences.

\section{ACKNOWLEDGMENT}

Tianyi Liu is thanked for her take part in the discussion of method implementation. This work is supported by the XXXXXXXXXXXXXXXXXXXXXXXXXXXXXXXXX.

Thanks to RoNIN to provide the data collection software for tango devices.

\section{REFERENCES}

[1] Y. Zhu, X. Luo, S. Guan, and Z. Wang, "Indoor Positioning Method Based on WiFi/Bluetooth and PDR Fusion Positioning," in 2021 13th International Conference on Advanced Computational Intelligence (ICACI), pp. 233-238.

[2] L. Zhang, Z. Chen, W. Cui, B. Li, C. Chen, Z. Cao, and K. Gao, "WiFiBased Indoor Robot Positioning Using Deep Fuzzy Forests," vol. 7, no. 11, pp. 10773-10781.

[3] F. Liu, J. Liu, Y. Yin, W. Wang, D. Hu, P. Chen, and Q. Niu, "Survey on WiFi-based indoor positioning techniques," vol. 14, no. 9, pp. 1372-1383. [Online]. Available: https://ietresearch.onlinelibrary.wiley. com/doi/abs/10.1049/iet-com.2019.1059

[4] H. Cao, Y. Wang, J. Bi, S. Xu, M. Si, and H. Qi, "Indoor Positioning Method Using WiFi RTT Based on LOS Identification and Range Calibration," vol. 9, no. 11, p. 627. [Online]. Available: https://www.mdpi.com/2220-9964/9/11/627

[5] Y. Wang and X. Li, "An improved robust EKF algorithm based on sigma points for UWB and foot-mounted IMU fusion positioning," vol. 66 , no. 2 , pp. 329-350. [Online]. Available: https://doi.org/10. 1080/14498596.2019.1632754

[6] A. I. Mourikis and S. I. Roumeliotis, "A Multi-State Constraint Kalman Filter for Vision-aided Inertial Navigation," in Proceedings 2007 IEEE International Conference on Robotics and Automation. IEEE, pp. 35653572. [Online]. Available: http://ieeexplore.ieee.org/document/4209642/

[7] T. Qin, P. Li, and S. Shen, "VINS-Mono: A Robust and Versatile Monocular Visual-Inertial State Estimator," vol. 34, no. 4, pp. 1004-1020. [Online]. Available: https://ieeexplore.ieee.org/document/ 8421746

[8] J. Kuang, X. Niu, and X. Chen, "Robust Pedestrian Dead Reckoning Based on MEMS-IMU for Smartphones," vol. 18, no. 5, p. 1391. [Online]. Available: https://www.mdpi.com/1424-8220/18/5/1391
[9] H. Yan, Q. Shan, and Y. Furukawa, "RIDI: Robust IMU Double Integration," in Computer Vision - ECCV 2018, ser. Lecture Notes in Computer Science, V. Ferrari, M. Hebert, C. Sminchisescu, and Y. Weiss, Eds. Springer International Publishing, vol. 11217, pp. 641-656. [Online]. Available: http://link.springer.com/10.1007/ 978-3-030-01261-8_38

[10] S. Herath, H. Yan, and Y. Furukawa, "RoNIN: Robust Neural Inertial Navigation in the Wild: Benchmark, Evaluations, New Methods," in 2020 IEEE International Conference on Robotics and Automation (ICRA), pp. 3146-3152.

[11] C. Chen, X. Lu, A. Markham, and N. Trigoni. IONet: Learning to Cure the Curse of Drift in Inertial Odometry. [Online]. Available: http://arxiv.org/abs/1802.02209

[12] S. Sun, D. Melamed, and K. Kitani. IDOL: Inertial Deep OrientationEstimation and Localization. [Online]. Available: http://arxiv.org/abs/ 2102.04024

[13] W. Liu, D. Caruso, E. Ilg, J. Dong, A. I. Mourikis, K. Daniilidis, V. Kumar, and J. Engel. TLIO: Tight Learned Inertial Odometry. [Online]. Available: http://arxiv.org/abs/2007.01867

[14] X. Ding, X. Zhang, J. Han, and G. Ding. RepMLP: Re-parameterizing Convolutions into Fully-connected Layers for Image Recognition. [Online]. Available: http://arxiv.org/abs/2105.01883

[15] H. Touvron, P. Bojanowski, M. Caron, M. Cord, A. El-Nouby, E. Grave, A. Joulin, G. Synnaeve, J. Verbeek, and H. Jégou. ResMLP: Feedforward networks for image classification with data-efficient training. [Online]. Available: http://arxiv.org/abs/2105.03404

[16] I. Tolstikhin, N. Houlsby, A. Kolesnikov, L. Beyer, X. Zhai, T. Unterthiner, J. Yung, D. Keysers, J. Uszkoreit, M. Lucic, and A. Dosovitskiy. MLP-Mixer: An all-MLP Architecture for Vision. [Online]. Available: http://arxiv.org/abs/2105.01601

[17] S. Roumeliotis and J. Burdick, "Stochastic cloning: A generalized framework for processing relative state measurements," in Proceedings 2002 IEEE International Conference on Robotics and Automation (Cat. No.02CH37292), vol. 2. IEEE, pp. 1788-1795. [Online]. Available: http://ieeexplore.ieee.org/document/1014801/

[18] A. Paszke, S. Gross, F. Massa, A. Lerer, J. Bradbury, G. Chanan, T. Killeen, Z. Lin, N. Gimelshein, L. Antiga, A. Desmaison, A. Kopf, E. Yang, Z. DeVito, M. Raison, A. Tejani, S. Chilamkurthy, B. Steiner, L. Fang, J. Bai, and S. Chintala, "PyTorch: An Imperative Style, HighPerformance Deep Learning Library," p. 12.

[19] D. P. Kingma and J. Ba. Adam: A Method for Stochastic Optimization. [Online]. Available: http://arxiv.org/abs/1412.6980

Xiaoji Niu received the B.Eng. degree (with honors) in Mechanical and Electrical Engineering and the Ph.D. from Tsinghua University, Beijing, China. He was a Postdoctoral Fellow with the Mobile MultiSensor Systems (MFMSS) Research Group, Department of Geomatics Engineering, and the University of Calgary. He was a Senior Scientist with SiRF Technology, Inc. At present, he is a Professor of the GNSS Research Center and the Collaborative Innovation Center of Geospatial Technology at Wuhan University, Wuhan, China. His research interests focus on INS, GNSS/INS integration for land vehicle navigation, and pedestrian navigation.

Jian Kuang received the B.Eng. degree and Ph.D. degree in Geodesy and Survey Engineering from Wuhan University, Wuhan, China, in 2013 and 2019, respectively. He is currently a Postdoctoral Fellow with the GNSS Research Center in Wuhan University, Wuhan, China. His research interests focus on inertial navigation, pedestrian navigation, and indoor positioning.

Yan Wang received the B.Eng. degree in Chemical Engineering and Technology from China University of Mining and Technology, Xuzhou, China, in 2016. And he received an M.S. degree in Computer Applied Technology from China University of Mining and Technology, Xuzhou, China, in 2019. $\mathrm{He}$ is currently pursuing a Ph.D. degree in GNSS Research Center, Wuhan University, Wuhan, China. His research interests focus on indoor navigation, sensor fusion algorithm, and computer vision. 